\title{
CONVIVENCIA Y CLIMA ESCOLAR: CLAVES DE LA GESTIÓN DEL CONOCIMIENTO
}

MARIO SANDOVAL MANRÍQUEZ*

\begin{abstract}
RESUMEN
El presente artículo aborda la temática de la gestión del conocimiento de los/las estudiantes y su relación con la convivencia y el clima escolar. Se aborda la escuela como un espacio de socialización secundaria, teniendo como materia prima de análisis el conjunto de interacciones sociales que se dan en su interior. La hipótesis de base que sustenta el presente trabajo es que en la medida que la convivencia escolar se verifique en un clima de respeto y tolerancia, sustentada en valores como la cooperación y la confianza, la gestión del conocimiento - entendido como un proceso donde los conocimientos y comunicaciones son orientados hacia un objetivo con habilidad y sabiduría - será más eficiente, redundando en mejores aprendizajes, contribuyendo con ello a mejorar la calidad de la educación.
\end{abstract}

\section{PALABRAS CLAVE: ESTUDIANTES, CONVIVENCIA ESCOLAR, CLIMA DE AULA, GESTIÓN DE CONOCIMIENTO}

* Doctor en Sociología (UCL), Coordinador del Centro de Estudios en Juventud de la Universidad Católica Silva Henríquez.

E-Mail: msandoval@ucsh.cl.

El presente artículo es producto del Proyecto de Investigación Fondecyt No1130449 «Clima escolar y gestión del conocimiento en la educación secundaria. Hacia una política de calidad pedagógica para la institución educativa en Chile». 


\title{
CONVIVÊNCIA E CLIMA ESCOLAR: CHAVES DA GESTÃO DO CONHECIMENTO
}

\begin{abstract}
RESUMO
O presente artigo abrange a temática da gestão do conhecimento dos e das estudantes e sua relação com a convivência e o clima escolar. No texto se compreende a escola como um espaço de socialização secundária, onde a origem da análise é o conjunto de interações sociais que acontecem em seu interior. A hipótese de base que sustenta o presente trabalho é que na medida em que a convivência escolar é verificada em um clima de respeito e tolerância, sustentada em valores como a cooperação e a confiança, a gestão do conhecimento, entendido como um processo onde os conhecimentos e comunicações são orientados para um objetivo com habilidade e sabedoria, será mais eficiente, resultando em melhores aprendizagens, contribuindo com isto para melhorar a qualidade da educação.
\end{abstract}

PALAVRAS CHAVE: ESTUDANTES, CONVIVÊNCIA ESCOLAR, CLIMA DE AULA, GESTÃO DO CONHECIMENTO

\section{COEXISTENCE AND SCHOOL ENVIRONMENT KNOWLEDGE MANAGEMENT KEYS}

\begin{abstract}
This article addresses the idea of how knowledge is managed by male and female students, as well as how they relate to each other and to their school environment. School is understood as a space of secondary socialization, being the set of social interactions that are held within it the main information source for the analysis. The main hypothesis that is held in this work is that, when school life is lived under the idea of respect and tolerance, and sustained under values such as cooperation and trust, knowledge management, understood as a process where knowledge and communication are oriented towards an objective with aptitudes and wisdom, will be more efficient, thus materializing in a better learning process, ultimately contributing in improving the overall quality of education.
\end{abstract}

KEY WORDS: STUDENTS, SCHOOL COEXISTENCE, KNOWLEDGE MANAGEMENT, SCHOOL ENVIRONMENT 


\section{INTRODUCCIÓN}

EN LAS RELACIONES SOCIALES, dentro y fuera del aula, se encuentran mundos diversos sustentados en experiencias y vivencias personales, alimentados por capitales culturales que interactúan recíprocamente; en suma, se produce una amalgama entre la cultura nacional, local, de las familias y de la institución educativa. Esta interacción socioeducativa es el núcleo del trabajo pedagógico. Allí se encuentran y relacionan personas condicionadas por experiencias distintas, socializadas en contextos disímiles, (F. Dubet, 1998) adhiriendo a modelos culturales contrapuestos (Bajoit, 1995): por una parte, los profesores/as (adultos) y por otra, los/las estudiantes (niños/jóvenes); allí se verifica la convivencia escolar en un determinado clima de aula y laboral. Lo tradicionalmente esperable es que cuando se produce esa interacción social al interior del aula, los profesores enseñen y los estudiantes aprendan, los profesores/as usen metodologías de enseñanza aprendidas en la universidad $-\mathrm{y}$ en las prácticas pedagógicas - y los estudiantes se motiven, haciendo precisamente lo que se espera de ellos: estudiar.

Sin embargo, la época actual que nos toca vivir, nos demuestra que actualmente son múltiples y diversas las fuentes de conocimiento a las que acuden las nuevas generaciones de estudiantes; es decir, no solo aprenden de las relaciones pedagógicas con sus maestros, sino que utilizan las nuevas tecnologías con una maestría envidiable, maestría que los profesores (adultos) manejan con dificultad o simplemente no tienen, así como también aprenden de sus pares, en sus zonas de desarrollo próximo, como diría Vygostsky (1988).

En este nuevo contexto, la escuela mantiene una misión aceptada socialmente: educar. En ese proceso se conjugan contenidos y valores que son considerados útiles para el desempeño social de los niños y jóvenes. A pesar de todos los cambios experimentados en las últimas décadas se sigue esperando que la escuela (los profesores/as) les enseñen a sus estudiantes y a su vez los/las estudiantes, aprendan de ellos/as. Sin embargo, un análisis profundo de esta interacción educativa contemporánea permite preguntarse:

- ¿Cómo aprenden los/las jóvenes estudiantes del siglo XXI?

- ¿Qué herramientas pedagógicas, didácticas y tecnológicas utilizan para gestionar su conocimiento?

- ¿Qué importancia tiene en ese proceso la convivencia y el clima imperante en la escuela? 
- ¿Estamos viviendo un cambio epistemológico en el paradigma educativo que tradicionalmente ha relacionado a profesores y estudiantes?

Es sabido que detrás de los contenidos y valores que se expresan en objetivos educacionales, entiéndase como «currículum explícito» simultáneamente existe una forma tácita de enseñanza que entrega normas sociales y expectativas que tiene la sociedad sobre los/las estudiantes. Es lo que Jackson (2001) llama «currículum oculto»; es decir, que las escuelas hacen lo que se supone que deben hacer; dicho de otra forma, entregan lo que será útil para el orden social establecido (Apple \& King, 1977).

A través del currículum oculto se transmiten las expectativas asociadas al papel de los/las estudiantes (sumisión, obediencia, etcétera), las cuales entran en contradicción con los objetivos del currículum explícito (autonomía, capacidad crítica), expectativas que con frecuencia resultan imposibles de entender para determinados estudiantes, a los que con frecuencia se denomina como «conflictivos».

Jackson (2001) destaca tres aspectos básicos del currículum oculto existente en la escuela tradicional que conducen al aprendizaje de la sumisión y la heteronomía:

La monotonía de la vida escolar. Los/las estudiantes deben aprender a esperar, a tener paciencia, a permanecer inmóviles, durante largos periodos de tiempo, ignorando a sus compañeros.

La naturaleza de la evaluación educativa: Su carácter no explícito, sus contradicciones y la imposibilidad de que los estudiantes puedan discutirla. Los/las estudiantes deben aprender cómo funciona este complejo mecanismo para garantizarse el máximo de recompensas y el mínimo de castigos, aprender a acomodarse a las expectativas de los demás y conseguir la aprobación simultánea de dos audiencias, el profesor y los compañeros, que con frecuencia entran en conflicto.

La fuerte jerarquización de la vida escolar y la concentración del control en el profesor: Los/las estudiantes deben acostumbrarse a la diferencia de poder, sustituyendo los propios planes e iniciativas por los que el profesor (a) impone.

El currículum oculto (Jackson, 2001) permite que se cree una cultura escolar determinada, fortalecida a través de prácticas propias de cada escuela, colegio o liceo, contenidas en el Proyecto Educativo Institu- 
cional — en adelante PEI - que refuerzan lo establecido, transmitiendo un sello identitario institucional, debiendo ejercitarlo en todos los espacios de aprendizaje escolar.

$\mathrm{Al}$ respecto, Magendzo (2006), señala que «el currículum oculto, es parte integral de la cultura escolar; se constituye en el filtro por el cual necesariamente llega la propuesta curricular a la escuela, se asienta en las relaciones interpersonales entre los docentes y estudiantes, entre estos y sus pares, en las relaciones jerárquicas de poder que priman entre directivos, docentes de aula, personal apoyo y también en forma muy importante en la relación que la institución escolar establece con los padres de familia».

De la misma forma que hay una distribución desigual del capital económico en la sociedad, también existe una distribución inequitativa del capital cultural. Es así como resurge la idea planteada por P. Bourdieu (1997) de la «reproducción de la desigualdad social» que se da en las escuelas, cuyo fundamento es la reproducción de las clases sociales con sus costumbres y modos de vida; con sus habitus, diría P. Bourdieu. Cuando se analiza el status-quaestionis del sistema educacional chileno y se constata la tremenda segmentación del mismo, no cabe más que darle la razón a este autor.

Sin embargo, sería reductivista plantear que el rol de la escuela se agota en la reproducción de la desigualdad social, porque también existen cambios en la sociedad. En concreto estamos viviendo un profundo proceso de cambio de modelo cultural (Bajoit, 2003); en consecuencia, si se aceptan ambas ideas, se ha de aceptar que la escuela también produce/reproduce esos cambios socioculturales; es decir, las actuales generaciones de estudiantes están aprendiendo envueltos en un proceso de profundo cambio cultural, que no terminan de comprender, ni asimilar.

En este contexto de cambio sociocultural acelerado, los/las profesores transmiten a las nuevas generaciones todo un acervo cultural con el fin de que los/las estudiantes se apropien de este para producir nuevas ideas y conceptos que harán reaccionar a la sociedad desde el punto de vista socioepistemológico, por ello, se han institucionalizado políticas educativas que propenden a mejorar el estado de la educación, al punto que hoy se habla de «la calidad de la educación».

Para ello, el aparato institucional educativo ha establecido formas de conocer y reconocer el estado del proceso educacional, a través de sistemas de evaluación, tanto para los/las estudiantes como también para los profesores/as, quienes ponen en movimiento la máquina de la institución educativa. 
Sin embargo, de facto, las evaluaciones son totalizadoras contemplándose el funcionamiento global de un proyecto curricular (L. Stenhouse, 1997), perdiéndose la individualidad de los actores principales de la trama educativa y desconociendo los aprendizajes que los jóvenes estudiantes obtienen de fuentes no tradicionales, o alternativas. por decirlo de alguna forma.

Ahora bien, en la práctica, así como en la teoría, el concepto de evaluación ha sufrido los rigores de una interpretación estrecha y simplista. La evaluación considera el énfasis en los resultados (SIMCE y PSU), con el olvido del análisis y valoración de los procesos de enseñanza, la primacía de los resultados observables, previamente establecidos como objeto de evaluación, no considerando la importancia de los efectos secundarios e imprevistos de todo proceso de enseñanza y la importancia de los significados y procesos cognitivos internos (Gimeno, 1989).

Son dos mundos distintos, por tratarse de dos actores diferentes, sin embargo, a diario, ambos actores - profesores y estudiantesconcurren a las aulas, donde se lleva a cabo el proceso de educación formal. Es allí donde interactúan estos sujetos socioculturales, entrelazando historias de vida, experiencias vitales, valores y expectativas, dando vida a un proceso de socialización secundaria (Dubet, 1998); por lo tanto, la convivencia y clima al interior del aula $-\mathrm{y}$ fuera de ella- juega un rol central en la gestión del conocimiento de los/las estudiantes, permaneciendo de fondo las preguntas antes señaladas: ¿cómo aprenden los estudiantes actuales? y ¿cuáles son las principales variables que inciden en sus aprendizajes?

Una línea de respuesta está dada por aquellos autores que colocan el énfasis en el capital cultural de las familias; es así como desde la década del 60 se han realizado varios estudios que han demostrado la influencia del «capital cultural» sobre el rendimiento de los/las estudiantes, particularmente de enseñanza básica y media, (Coleman et al., 1966; Mizala, Romanguera y Reinaga, 1999; Díaz et al., 2002; Decouvières et al., 2005; Apablaza et al., 2009; Díaz y Leighton, 2010). Estas investigaciones han demostrado claramente la importancia del llamado «efecto cuna», concepto que reúne una serie de factores predisponentes que emanan desde el hogar y por consiguiente, de las familias (J. J. Brunner, 2010).

Para otros investigadores como Casassus (2003); Hanushek y Rivkin (1997), es la escuela y el aula, como espacio físico, lo que favorecen el aprendizaje a causa del «clima de aula» afectivo que se obtiene a través de la interacción pedagógica, donde los profesores/as 
juegan un rol fundamental, puesto que el resultado obtenido por los/las estudiantes dependerá directamente de las acciones pedagógicas que dicho docente realice para el logro de un clima de aula favorable al aprendizaje; dicho de otro modo: en la medida que los profesores/as logren en el aula un clima de tranquilidad, relajación y confianza, sus estudiantes van a aprenden más y mejor.

En ese contexto, el «Marco para la buena enseñanza» del MINEDUC (2008) reconoce la importancia de la convivencia y clima escolar en la gestión del conocimiento - en particular el punto 2-, al señalar que este Marco está conformado por cuatro dominios:

- Preparación de la enseñanza.

- Preparación de un ambiente propicio de aprendizaje.

- Enseñanza para el aprendizaje de todos los estudiantes.

- Responsabilidades profesionales.

Dicho lo anterior, debemos reconocer que la escuela es un sistema organizado, institucionalizado y jerarquizado, cuyo fin es que los/las estudiantes conozcan, aprendan y manejen herramientas para desenvolverse en la sociedad, considerándola a esta como «una organización con un marcado acento en la satisfacción de necesidades intelectuales y académicas» (Arancibia, Herrera y Strasser, 1999).

Al mismo tiempo se espera que los/las estudiantes desarrollen habilidades y competencias en las que sustenten su realización personal, su inclusión social, su formación para la ciudadanía y su capacitación para gestionar, de forma adecuada, su subjetividad en el marco del respeto a los demás.

Considerando lo anterior, Oliva, Montes y Torrellas (2009), señalan que «los retos actuales a los que se enfrentan las instituciones educativas son complejos, pues hay que trabajar en varias direcciones que permitan simultáneamente incrementar la equidad, la eficiencia de las instituciones como formadoras del ser e incrementar su calidad y pertinencia» (p. 163). Como fue dicho anteriormente, la convivencia y el clima escolar juegan un rol fundamental en la gestión del conocimiento de los establecimientos educacionales, es por ello que a continuación desarrollaremos estos tres conceptos. 


\section{CONVIVENCIA ESCOLAR}

La convivencia escolar es un elemento fundamental en un proceso escolar satisfactorio y productivo. Esta premisa constituye el fundamento de un conjunto de iniciativas políticas, legislativas y programáticas desplegadas en Chile por diferentes gobiernos desde los años 90 del siglo pasado, reconociendo implícitamente el rol que esta juega en la gestión del conocimiento de los/las estudiantes.

Las políticas educativas han asumido este desafío fomentando la participación integral de las comunidades educativas, promoviendo la constitución de Centros de Padres y Apoderados, incentivando la formación de Centros de Alumnos, concibiéndolos como organizaciones que canalizan esta participación. La convicción de las autoridades educativas es que si la convivencia escolar es adecuada esto redundará en una mejora de los aprendizajes.

La Ley $\mathrm{N}^{\circ} 20.536$ sobre violencia escolar, publicada el 17 de septiembre de 2011, define la convivencia escolar como la «coexistencia armónica de los miembros de la comunidad educativa, que supone una interrelación positiva entre ellos y permite el adecuado cumplimiento de los objetivos educativos en un clima que propicia el desarrollo integral de los estudiantes» (Ley sobre Violencia Escolar, párrafo 3, 2011).

En este sentido, se entiende por «convivencia» la potencialidad que tienen las personas para vivir con otros, en un marco de respeto mutuo y de solidaridad recíproca. La convivencia escolar se genera en la interrelación entre los diferentes miembros de la comunidad educativa de un establecimiento educacional que tiene incidencia significativa en el desarrollo ético, socioafectivo e intelectual de los niños y jóvenes.

Dicha concepción no se limita solo a la relación entre las personas, sino que incluye las formas de interacción entre los diferentes estamentos que conforman la comunidad educativa, por lo que constituye una construcción colectiva y es responsabilidad de todos quienes participan del proceso educativo - profesores, estudiantes, padres y apoderados, asistentes de la educación-.

Vivir y convivir con otros en armonía supone el respeto a las ideas y sentimientos de los demás, la tolerancia frente a las diferencias, la aceptación y valoración de la diversidad, la solidaridad, reciprocidad y cooperación mutua en función de objetivos comunes.

La convivencia escolar tiene un enfoque formativo, por lo mismo se considera la base de la formación ciudadana y constituye un factor clave de la formación integral de los/las estudiantes; los profesores/as 
deben enseñar y los/las estudiantes aprender una suma de conocimientos, habilidades, valores y principios que les permitan poner en práctica el vivir en paz y armonía con otros; es por eso que la gestión formativa de la convivencia escolar constituye la forma más efectiva de prevención de la violencia escolar.

Dado que la experiencia/vivencia de la convivencia escolar es un espacio/momento de formación de ciudadanía no solo involucra a los/las estudiantes sino que a todos los integrantes de la comunidad educativa; debiendo ser ejercitada por los profesores/adultos, para ser enseñada, aprendida y ponerla en práctica en todos los espacios formales e informales de la vida escolar.

Una convivencia escolar sana, armónica, sin violencia, incide directamente en la calidad de vida de todos los miembros de la comunidad educativa, en los resultados de los aprendizajes, en la gestión del conocimiento y en el mejoramiento de la calidad de la educación. Relacionarse con otros en paz es el fundamento de una convivencia social democrática, la cual se constituye en un aprendizaje que debe ser intencionado desde las prácticas pedagógicas, tanto en el aula como fuera de ella, asumiéndola como una tarea educativa/formativa que es de responsabilidad de todos los miembros de la comunidad escolar.

Para lograr esta convivencia social pacífica y democrática entre los miembros de la comunidad educativa, es necesario el aprendizaje y la práctica de valores como el respeto, la solidaridad, la ayuda mutua - entre otros - y el desarrollo de la capacidad de las personas para convivir en armonía. En ese sentido, la tarea de educar en valores no está circunscrita solo al ámbito escolar; la familia y la sociedad son espacios sociales fuertemente comprometidos en esta responsabilidad.

Hay una primera concesión de esta amplia responsabilidad que afecta a la persona del educador. Si los/las profesores en la escuela han de contribuir a que los/las estudiantes se descubran a sí mismos, descubran el mundo y su profundo significado, no es indiferente el concepto de ser humano y de mundo que tengan. Y más que el concepto, más que la visión intelectual, importa su actitud valorativa de las demás personas y de su inserción en el mundo; lo que él sea y el modo, incluso, de autoconocerse, constituye el aporte fundamental al proceso de autorrealización de los niños y jóvenes, de su relación con los demás, con el entorno que lo rodea, con el medio ambiente y en consecuencia, la actitud que tengan frente a los derechos humanos, en su más amplia acepción. 
Pero la educación no se reduce a la relación profesor-alumno. En el marco de la escuela como institución se da una interacción constante entre la estructura, la organización y la metodología didáctica. Estos conllevan a juicios de valor y convierten a estos medios en vehículos decisivos de esquemas de valoración y de adhesión a determinados valores.

La educación en valores es una de las áreas educativas más interesantes y más conflictivas, puesto que es un campo que exige una profunda reflexión y discusión y sobre la cual todavía no hay acuerdos en el mundo educacional. Como respuesta a esta necesidad percibida con urgencia por algunos profesores/as, han surgido diversas corrientes y métodos bajo el nombre genérico de «educación humanista». Este tema ha atraído el interés de profesores/as, estudiantes, psicólogos, sociólogos y filósofos.

A pesar de esta corriente de búsqueda en amplios sectores educacionales, tenemos que reconocer que el tema está en periodo de gestación, y aun los mismos conceptos de «valor» y «valoración» están en proceso de ser clarificados para llegar a un lenguaje común más o menos aceptado de manera universal.

Superka (1973) —en Santoyo, 2005—, elaboró una tipología para una educación en valores, considerando cinco áreas fundamentales:

Inculcación: El objetivo de este enfoque es infundir o internalizar determinados valores que son considerados como deseables. Si el sujeto de la educación (niño o joven) ha de ser el protagonista de su libertad hay que considerar que sea él/ella quien descubra los valores y opte libremente por ellos.

Son varios los métodos utilizados para la inculcación de valores: el del refuerzo y propuesta de modelos. La combinación del refuerzo con la propuesta de modelos es un medio para inculcar valores. Si el modelo es reforzado positivamente, los observadores de la conducta presentada como modelo tienen más probabilidades de actuar de modo semejante y, en consecuencia, de adoptar ese valor. Esta estrategia puede utilizarse intencionada y sistemáticamente para inculcar valores deseables.

Desarrollo moral: Este enfoque se basa en los postulados Piaget (1934, 1964) y Kohlberg $(1976,1997)$ y se ocupa del desarrollo cognitivo estimulando a los/las estudiantes para que sean capaces de desarrollar modelos más complejos de razonamiento moral a través de pasos secuenciales. 
La teoría del desarrollo moral utilizada por Kohlberg (1976) se describe en tres niveles y seis pasos:

\begin{tabular}{|l|l|}
\hline NIVELES & PASOS \\
\hline Preconvencionales & $\begin{array}{l}\text { Orientación al castigo y la vigilancia. } \\
\text { Orientación relativista instrumental. }\end{array}$ \\
\hline Convencionales & $\begin{array}{l}\text { Orientación a la conformidad interpersonal. } \\
\text { Orientación a la ley y el orden. }\end{array}$ \\
\hline Postconvencionales & $\begin{array}{l}\text { Orientación legalista hacia el control social. } \\
\text { Orientación al principio ético universal. }\end{array}$ \\
\hline
\end{tabular}

Estos niveles se corresponden con los consiguientes pasos que marcan un proceso de desarrollo moral. Kohlberg (1976) parte de una orientación en desarrollo, y plantea que es necesario seguir una secuencia de pasos. Este autor indica que el exponer a los niños y jóvenes a niveles superiores de razonamiento los estimula a alcanzar el paso siguiente superior del desarrollo moral. Esta teoría aporta un aspecto importante a la educación de valores.

a) Análisis: las técnicas de análisis constituyen un enfoque de la educación en valores elaborado por educadores del campo de las ciencias sociales. El objetivo de esta técnica es ayudar a los/las estudiantes a usar el planteamiento lógico y los procedimientos de investigación científica relativos a los valores. Ellos deben aportar hechos verificables acerca de la validez de los fenómenos. Su aporte consiste en ofrecer una base sólida para llegar a hacer opciones razonables, teniendo en cuenta la base objetiva que ofrece. Los pasos de este método son los siguientes:

- Identificar y clarificar la cuestión del valor.

- Recoger los hechos significativos.

- Evaluar la veracidad de los hechos recogidos.

- Clarificar la relevancia de los hechos.

- Llegar a una primera decisión valorativa provisional.

- Medir el principio de valoración implicado en la decisión.

b) Clarificación de valores: esta técnica es una de las más famosas y extendidas, desarrollada por el profesor Sidney Simon (1995) de la Universidad de Massachusetts. El objetivo es ayudar a los/las estudiantes a tomar contacto con aquello que actualmente constituye un valor en sus vidas y ayudar a la persona a descubrir la realidad de su orientación, de sus ideas. El fin es afianzar estos valores una vez reconocidos y aceptados, o de cambiarlos si carecen de consistencia. Este proceso implica tres momentos fundamentales: 
Elección libre: para que el niño/joven llegue a ser un elemento constitutivo de su «yo».

Estimación: para que la valoración sea real, debe producirle satisfacción y disfrutarla.

Coherencia en la acción: para que pueda considerarse que hay un valor presente, la vida misma debe ser afectada por él/ella.

Estos tres momentos en el proceso de valoración se desglosan en siete pasos que son considerados imprescindibles para que algo pueda considerarse como un valor.

i) Escoger libremente los valores: deben ser espontáneos y libres de elección.

ii) Escoger los valores entre distintas alternativas: las alternativas deben guardar relación entren sí, ser formuladas para que puedan entenderse con facilidad y así surja un valor.

iii) Escoger los valores después de sopesar las consecuencias de cada alternativa: hay que medir el peso axiológico de cada una de las posibilidades que se ofrecen.

iv) Apreciar y estimar los valores: cuando concedemos valor a una cosa la apreciamos, la disfrutamos, la estimamos, la respetamos y la queremos.

v) Compartir y afirmar públicamente los valores: cuando elegimos algo libremente, lo analizamos y sentimos alegría, no vacilamos en afirmar nuestra decisión.

vi) Actuar de acuerdo con los propios valores: es preciso que la conducta afirme nuestras decisiones y las integre en la vida.

vii) Actuar de acuerdo con los propios valores de una manera repetida y constante: los valores tienden a ser persistentes, a dar forma a la vida humana. No se podría considerar valor algo que aparece una vez en la vida y que no vuelve a presentarse.

La clarificación de valores es un proceso progresivo personal que abarca toda la vida. A medida que el mundo cambia y nosotros mismos cambiamos, aparecen muchas decisiones a tomar y es importante aprender la forma más adecuada de asumir las decisiones. Este método persigue únicamente que el individuo se haga consciente de sus propios valores. Que esté inicialmente estimulado para comenzar la búsqueda y el adiestramiento en unos valores que le den sentido a su vida y que lo conduzcan al proceso de convertirse en persona.

Aprendizaje para la acción: su objetivo es proporcionar a los/las estudiantes oportunidades específicas para actuar según sus valores, dentro y fuera del aula. Las técnicas de aprendizaje para la acción consideran a la persona fundamentalmente como interactiva. En este modelo se en- 
cuentran los primeros grados de desarrollo, que están incluidos en los seis pasos siguientes:

- Tomar conciencia del asunto o proceso.

- Comprender el asunto o problema y tomar una postura.

- Decidir una actuación.

- Planificar estrategias y etapas para la acción.

- Aplicar actividades y realizaciones de la acción.

- Reflexionar sobre las acciones emprendidas y considerar los pasos siguientes (las consecuencias).

El aporte fundamental de esta técnica es que busca llevar al niño/joven a comprometerse activamente con los valores estimados como tales por él/ella mismo/a. Trata de poner al sujeto en situaciones concretas que lo inciten a comportarse de acuerdo con sus propios valores.

Como fue señalado anteriormente, una convivencia escolar sana y armónica debe basarse en valores como el respeto, la tolerancia, la diversidad y debe reunir, al menos, las siguientes características:

- La convivencia escolar es la relación entre todos los actores institucionales. Esto implica que los niños, jóvenes y adultos son considerados partícipes de la convivencia, adscribiéndoseles derechos y responsabilidades, tal como plantea la Política de Convivencia Escolar del Ministerio de Educación chileno (2001).

- La convivencia escolar se vive a diario, siendo expresión de lo que cada persona es, refiriendo códigos y maneras aprendidas en los contextos de convivencia.

De acuerdo con la Ley de Violencia Escolar (2011), el aprendizaje de la convivencia escolar debería ser intencionado y modelado por los/las profesores, cuyos contenidos se encuentran en los Objetivos Fundamentales Transversales de los diferentes subsectores de aprendizaje, cuyo propósito es desarrollar las siguientes habilidades:

- Aprender a interactuar: intercambiar acciones con otros/as.

- A interrelacionarse, establecer vínculos que implican reciprocidad.

- A dialogar con fundamentos.

- A escuchar activamente y hablar con otros/as.

- A participar: actuar con otros/as.

- A comprometerse: asumir responsablemente las acciones con otros/as.

- A compartir propuestas.

- A discutir e intercambiar ideas y opiniones con otros/as. 
- A disentir; aceptar que mis ideas- o las del otro/a u otros/as-, puedan ser diferentes.

- A consensuar: encontrar los aspectos comunes, lo que implica pérdida y/o ganancia.

- A reflexionar: repensar sobre lo actuado, lo sucedido; objetivar y observar críticamente las acciones e ideas (Ianni, 2003).

De esta manera, se estaría adquiriendo el sello de un aprendizaje intencionado, siendo un importante eje para la formación de personas que aprenden una determinada manera de convivir en una convivencia pacífica y democrática.

Un contexto escolar participativo y democrático donde los sujetos tienen diversas oportunidades de ejercicio progresivo de sus derechos y consecuentes responsabilidades, posibilita aprehender, en convivencia con otros, el respeto al otro y la corresponsabilidad en la construcción del clima cooperativo necesario para aprender a ser, aprender a vivir juntos, aprender hacer y aprender a aprender (MINEDUC, 2005:189).

El aprendizaje de la convivencia escolar tiene una dimensión preventiva, ya que pretende formar en los/las estudiantes un pensamiento crítico y autónomo, con el fin de que tomen de decisiones personales responsables, anticipándose a situaciones que amenacen o alteren la convivencia pacífica con sus pares.

Los procesos cognitivos, afectivos, emocionales y sociales que se desarrollan en las aulas y fuera de ella, son los que van articulando el conjunto de hábitos, conductas, actitudes y valores que componen la personalidad de los niños y jóvenes y cuyo desarrollo óptimo favorece la construcción de personalidades críticas, sanas y equilibradas moralmente.

\section{a) Convivencia escolar y comunidad educativa}

La Comunidad Educativa es un espacio privilegiado para aprender a vivir con otras personas. La institución escolar, como espacio de formación, permite vivenciar el ejercicio de la vida democrática, preparando a los/las estudiantes para el diálogo social, cultural y político que se requiere en el mundo actual; por eso, también es el lugar donde se aprende la convivencia ciudadana (MINEDUC, 2011).

De esta manera, el Proyecto Educativo Institucional (PEI) se convierte en una guía orientadora que permite definir el marco conceptual, filosófico, valórico, pedagógico y de estilo de gestión educativa 
del establecimiento. La Misión y Visión que allí se declaran se convierten en un desafío y, a la vez, clarifican las formas de proceder, centrados en un servicio educacional de calidad, de excelencia, modélico y transferible, que exige un fuerte compromiso e identidad de quienes lo entregan y lo reciben.

Gairín y Antúnez (1999) no señalan que cualquier PEI debe responder a las siguientes preguntas:

- ¿Dónde estamos? Su respuesta explica el medio circundante, el marco legal, la reseña histórica, la procedencia y características propias del alumnado, los programas y redes de apoyo escolar del establecimiento.

- ¿Quiénes somos? Su respuesta explica los fundamentos antropológicos y axiológicos, los principios de identidad, marco valórico y el perfil de los/las estudiantes que se desean formar.

- ¿Qué queremos? Su respuesta explica las implicancias del Marco Pedagógico, los objetivos educacionales a conseguir, los lineamientos de trabajo, los planes de estudio y programas complementarios que desarrolla la comuna, las actividades extraprogramáticas propuestas, el perfil ideal del profesor(a) y los reconocimientos y premios a los/las estudiantes.

- ¿Cómo nos organizamos? Su respuesta explica la estructuración a seguir desde el ámbito superior constituido por la organización, pasando por el establecimiento educativo para llegar hasta la sala de clases.

En consecuencia, un PEI, debe responder a las siguientes necesidades:

- Identificar con claridad las características propias de identidad institucional, tanto en la oferta académica como en lo formativo, a la luz de las nuevas necesidades y objetivos planteados.

- Otorgar una intencionalidad determinada a la educación que se imparte en la escuela, colegio o liceo, definida en una búsqueda común de los/las estudiantes, profesores/as, padres y apoderados, alineados con la Misión y la Visión del establecimiento educacional.

- Ordenar y sistematizar el desarrollo del proceso educativo, colaborando con el cumplimiento de los objetivos y metas a corto, mediano y largo plazo, definidos por las autoridades.

- Entregar una educación de calidad, acorde a los lineamientos establecidos en el PEI, que permita satisfacer las expectativas de oferta educativa en coherencia con la Misión y Visión declaradas.

Es así como la comunidad escolar, a través de su PEI, debe expresar los intereses formativos de los/las estudiantes, de los padres, madres y 
apoderados/as, de los profesores/as, de los directivos y de los asistentes de la educación y, constituyéndose en una comunidad de intereses comunes, con objetivos compartidos.

La participación, la capacidad de escuchar y compartir opiniones hacen de este espacio un lugar de vivencia y de práctica democrática, donde «aprender a convivir» es un eje central. De allí que la responsabilidad por la convivencia escolar en el establecimiento educacional sea de todos quienes participan en ella. En la escuela los/las estudiantes aprenden sobre la vida y aprenden a convivir, siempre que este ambiente sea propicio para desarrollar sus capacidades, habilidades, competencias y talentos.

Al respecto, Maturana (2001) expresa que «es primordial enseñar a un niño/a a respetarse y aceptarse, solo así aprenderá a respetar y a aceptar a sus compañeros y vivir en armonía con su entorno» (Cid, Díaz y Pérez, 2008).

De esta manera se forman las redes de apoyo en la que los niños y jóvenes se involucran, compartiendo actividades, objetivos, ideas, sentimientos, emociones y valores, fortaleciendo su capital social. Las principales redes de apoyo de los jóvenes estudiantes están constituidas por sus familias, sus compañeros y compañeras de curso, por sus amigos y amigas, por sus vecinos y por las instituciones que frecuentan, contribuyendo con ello a su formación ciudadana.

Los jóvenes estudiantes del siglo XXI tienen el desafío de vivir en una sociedad multicultural e intercultural, constituyéndose en la base del aprendizaje de la convivencia social/escolar. Este aprendizaje tiene su origen en las familias (socialización primaria), y luego la escuela (socialización secundaria) debe reforzar dichos aprendizajes a través de su currículum explícito, así como en su currículum oculto.

Desde su tarea formativa, Soto (1995) plantea cuatro ideas fundamentales referidas a la escuela (en Redondo, 2009):

i) Las escuelas no pueden ser analizadas como instituciones separadas del contexto socioeconómico en el que están situadas.

ii) Las escuelas son sitios políticos involucrados en la construcción y control de discurso, significado y subjetividades.

iii) Los valores del sentido común y las creencias que guían y estructuran las prácticas en la sala de clases, no son universales a priori, sino que son construcciones sociales basadas en supuestos normativos y políticos específicos.

iv) La socialización dada en la escuela no es un simple vehículo de transmisión del orden social, sino que agencia de control social 
que funciona para ofrecer formas diferenciadas de enseñanza a diferentes clases sociales.

Relacionado con lo anterior, los actores educativos, deben ser capaces de crear una atmósfera agradable y segurizante para que se cultiven relaciones humanas basadas en una sana convivencia, creando una comunidad escolar, la que está definida en la Ley General de Educación como «una agrupación de personas que inspiradas en un propósito común, integran una institución educativa» (LEGE, Art. 8), cuyo objetivo es «contribuir a la formación y el logro de aprendizajes de todos los alumnos» y «asegurar su pleno desarrollo espiritual, ético, moral, afectivo, intelectual, artístico, y físico». Este propósito se expresa «en la adhesión al proyecto educativo del establecimiento y a sus reglas de convivencia establecidas en el reglamento interno» (LEGE, Art. 9).

\section{Clima escolar}

El desarrollo del concepto de clima escolar (Mena y Valdés, 2008) tiene como precedente el concepto de «clima organizacional», resultante del estudio de las organizaciones en el ámbito laboral, a partir de fines de la década del 60 (Tagiuri \& Litwin, 1968; Schneider, 1975).

Este concepto surge como parte del esfuerzo de la psicología social por comprender el comportamiento de las personas en el contexto de las organizaciones, aplicando elementos de la Teoría General de Sistemas (Rodríguez, 2004).

A esto se le ha denominado «clima escolar» y ha sido definido como la percepción y sensación que un individuo tiene a partir de sus experiencias en el sistema escolar. Se relaciona con el poder de retención (estudiantes y profesores/as), grado de satisfacción y calidad de la educación (Arón y Milicic, 2004), lo cual favorecería la enseñanza y el aprendizaje de la comunidad educativa.

El CERE (1993) define el clima escolar como «el conjunto de características psicosociales de un centro educativo, determinados por aquellos factores o elementos estructurales, personales y funcionales de la institución, que, integrados en un proceso dinámico específico, confieren un peculiar estilo a dicho centro, condicionante, a la vez de los distintos procesos educativos» (p. 30).

Mientras que Milicic (2001) lo define como la percepción que los miembros de la institución escolar tienen respecto del ambiente en el cual desarrollan sus actividades habituales. Tales percepciones, se 
basarían en la experiencia que el propio individuo desarrolla en la interacción.

Por su parte, Cornejo y Redondo (2001), señalan que el clima social escolar refiere a «la percepción que tienen los sujetos acerca de las relaciones interpersonales que establecen en el contexto escolar (a nivel de aula o de centro) y el contexto o marco en el cual estas interacciones se dan» (p. 6).

Una particularidad de las instituciones educativas es la complejidad de sus climas - de aula y laboral-, puesto es que a diferencia de la mayoría de las organizaciones, en las escuelas el destinatario de la finalidad de la organización es a la vez parte de ella. Su Misión institucional es la formación de personas — los/las estudiantes-, son parte activa de la vida de la organización (Casassus, 1993).

En este sentido, respecto a los procesos educativos, cabe señalar que para que estos puedan realizarse adecuadamente, es necesario visibilizar los factores que lo componen; entre ellos, el clima escolar; es así como la UNESCO (2000) plantea que «la posibilidad de que la escuela sea significada por el alumno como una experiencia emocionalmente positiva va a depender en gran medida del ambiente que logren crear los alumnos y los profesores en el contexto educacional» (UNESCO, 2000).

Desde otra perspectiva, Mertz (2006) plantea que la escuela «es el ámbito de intervención privilegiado para la socialización y formación de valores pro sociales, y para prevenir que los niños y jóvenes sean víctimas o autores de un amplio rango de conductas negativas, ahora y en el futuro» (p.16).

Las acciones de prevención del maltrato y la violencia en la escuela, deben considerar la formación en disciplina, concebida esta como un conjunto de sanciones enmarcadas en un proceso de crecimiento y formación progresivos - y no como meros castigos-, en el que los/las estudiantes van compartiendo objetivos; internalizando, apropiándose y ensayando los roles que desempeñarán en contextos sociales más amplios y de los que parten responsabilizándose en la escuela; esto es ni más ni menos que educación ciudadana.

La disciplina es un componente central del clima escolar. La disciplina entendida como el cumplimiento de los roles que a cada miembro de la comunidad educativa le compete. Por tanto, la indisciplina no debe ser vista exclusivamente en relación a los/las estudiantes, sino también respecto de los adultos que forman parte de la comunidad escolar: docentes, padres, madres y apoderados, directivos, etcé- 
tera. Cada miembro tiene responsabilidades que cumplir y dar cuenta a los demás para alcanzar una meta común.

De acuerdo a lo anterior, los establecimientos educacionales juegan un rol clave en la prevención de conductas de riesgo y/o de delitos. La efectividad de estas acciones preventivas dependerá de la gestión y liderazgo educativo, del concepto de disciplina incorporado en el Reglamento de Convivencia y del desarrollo de habilidades y competencias sociales en los/las estudiantes.

Grisay, (1993) señala que el clima escolar debe ser considerado como eje de comprensión, no solo de una estructura de comportamiento, sino como la forma en la cual la escuela configura su mundo educativo en relación con sus sentidos; y para la construcción de este concepto, menciona cinco criterios, que a su vez permiten medirlo:

i) Sentimiento de los alumnos de ser objeto de atención.

ii) Sentimiento de los alumnos de justicia y equidad.

iii) Sentimiento de los alumnos de competencia (de sentirse competente) y de capacidad.

iv) Sentimiento de orgullo de los alumnos por estudiar en esa escuela.

v) Sentimiento de satisfacción general de los alumnos por su escuela.

En un clima escolar positivo los acontecimientos de crisis son más fáciles de gestionar y presentan menos riesgo de tomar decisiones no deseadas (Gottfredson, 2001). En este sentido, una tarea clave de los/las profesores es enseñar a sus estudiantes a resolver conflictos de manera no violenta; desterrando la ilusión de que no van a haber conflictos, puesto que el conflicto es inherente al funcionamiento social.

\section{a) Microclimas en la escuela}

El clima social escolar se compone de diferentes microclimas, que pueden ser protectores u obstaculizadores del desarrollo organizacional, paralelamente al clima general imperante.

En una escuela en la que existe un clima general negativo, las personas envueltas en microclimas positivos pueden verse protegidas por estos (Arón y Milicic, 2004). Los microclimas pueden ir desde grupos de amigos al interior de un curso/escuela hasta un curso completo dentro del nivel o equipos deportivos, artísticos, agrupaciones religiosas o de trabajo comunitario, entre otros. Así mismo, se pueden encontrar dentro de la comunidad escolar microclimas en diferentes 
espacios (departamentos, salas de profesores, edades, género, etcétera) y amplitudes (pueden ir desde grupos muy reducidos hasta toda la comunidad docente).

Dentro de una organización escolar pueden distinguirse dos microclimas principales y determinantes del proceso de enseñanzaaprendizaje: el clima de aula y el clima laboral.

i) Clima de aula: un «clima de aula» favorecedor del desarrollo personal es aquel en que los/las estudiantes perciben apoyo y solidaridad de parte de sus pares y profesores/as, se sienten respetados en sus diferencias y falencias, así como identificados con el curso y su escuela. Además, sienten que lo que aprenden es útil y significativo (Ascorra, Arias y Graff, 2003); tienen una percepción de productividad, de una atmósfera cooperativa y de preocupación; sienten que sus profesores/as están centrados en sus necesidades y que existe una buena organización de la vida de aula (Johnson, Dickson y Johnson; en Arón y Milicic, 2004).

ii) Clima laboral: el «clima laboral» corresponde al medio humano y físico en el que se desarrolla el trabajo cotidiano de los/las profesores y otros trabajadores de la escuela. Se relaciona con la manera de trabajar y de relacionarse entre profesores/as, con la institución y con las autoridades. El clima laboral como grado de satisfacción y percepción de bienestar con el trabajo y la organización afecta fuertemente el desempeño docente $y$, por ende, repercute en su trabajo con los niños y jóvenes (Arón y Milicic, 2004; Vail, 2005; UNICEF, 2005; Rodríguez, 2004).

Uno de los efectos positivos de un clima laboral adecuado es que contribuye a una convivencia escolar armónica y relajada, generando profesores/as motivados, con sensación de bienestar con su trabajo, ayudando a todos sus estudiantes a lograr sus objetivos de aprendizaje.

\section{b) Evaluación del cluma social escolar}

Según Milicic (2001), evaluar el clima social de una organización es fundamental, ya que permite identificar los probables obstáculos del logro de los objetivos de la institución. Si bien son muchas las variables que pueden influir, considera cinco en la evaluación del clima de cualquier organización: 
- Estructura de la organización.

- Relaciones humana.

- Recompensas.

- Reconocimiento.

- Autonomía.

c) Estrategias de mejoramiento del clima social escolar

Cornejo y Redondo (2001) proponen seis ejes estratégicos para mejorar el clima social escolar de las aulas:

- Afectividad y relaciones interpersonales de mayor cercanía e intimidad.

- Incorporación de la(s) cultura(s) juveniles a la dinámica escolar.

- Sentido de pertenencia con la institución.

- Participación y convivencia democrática.

- Sensación de pertinencia del currículum escolar.

- Mejora del autoconcepto académico de los alumnos.

El estudio del clima en el contexto escolar ha arrojado resultados que muestran cómo este constructo se relaciona con aspectos tan relevantes como la capacidad de retención de las escuelas, el bienestar y desarrollo socio/afectivo de los niños y jóvenes, el bienestar de los profesores/as, el rendimiento y la efectividad escolar, entre otros. Resultados que han sido un aporte para la comunidad educativa de los distintos establecimientos educacionales, al permitirles generar propuestas a partir de ellos, en pro de un mejoramiento no solo en su gestión sino que también en sus relaciones interpersonales.

\section{GESTIÓN DEL CONOCIMIENTO}

Por gestión del conocimiento entendemos el proceso donde los conocimientos y comunicaciones son orientados hacia un objetivo con habilidad y sabiduría; en ese sentido podemos abordar la gestión desde la acción/perspectiva individual e institucional.

A nivel individual, la gestión del conocimiento supone una actitud constructiva y crítica hacia el medio ambiente que lo rodea, desarrollando destrezas versátiles para la gestión de la vida, creando y fomentado lazos de cooperación recíproca con los demás, incentivando el desarrollo de habilidades y competencias tecnológicas y profesionales. 
Las personas que gestionan adecuadamente el conocimiento se adaptan creativamente al desarrollo tecnológico, a la internacionalización de las comunicaciones y a la vida cívica; son operadores activos y creativos, curiosos e inquietos intelectualmente, innovadores en la búsqueda de soluciones a los problemas cotidianos de estudio y trabajo.

Un estudiante que gestiona adecuadamente el conocimiento no se conforma con las respuestas dadas por sus profesores/as, siempre busca más, lee más, organiza su tiempo constructivamente, siempre pregunta; se plantea desafíos y estudia/trabaja en red.

Si pudiéramos definir el perfil de un estudiante que gestiona adecuadamente el conocimiento lo podríamos resumir en los siguientes conceptos: independiente, autónomo, estudiante permanente, orientado a metas, buen comunicador, cooperador, innovador, creativo, multitarea, constante, disciplinado y proactivo.

A nivel institucional, una escuela que gestiona bien el conocimiento sostiene la premisa que no hay una sola solución correcta a los problemas; por el contrario, incorpora los distintos puntos de vista de los actores involucrados (profesores/as, estudiantes, padres y apoderados, asistentes de la educación).

La gestión del conocimiento de una escuela debe ser entendida desde las propias premisas, principios, valores, misión, visión y desafíos contenidos en el PEI.

Desde la escuela, la gestión del conocimiento enfatiza la naturaleza dinámica del progreso, el poder del cambio permanente y las actividades claramente orientadas hacia objetivos logrables, estas son organizaciones que aprenden (Gairín, 2008).

Desde esa perspectiva, las escuelas deben establecer una fuerte integración entre trabajo y aprendizaje, considerando para ello la experiencia, la historia local y la continua experimentación que encuentra sólidas bases en la reflexión conjunta de sus profesores/as; flexibilizando sus formas de dirección, teniendo presente la necesidad de contar con mayor participación, delegación, trabajo colaborativo, autonomía y libertad.

Lo anterior permite hablar de organizaciones inteligentes o de organizaciones que aprenden, ya que estas son definidas como aquellas que aumentan continuamente su capacidad para construir futuro, tienen habilidades para crear, adquirir y transferir conocimientos y saberes.

Las organizaciones que aprenden vienen a significar; por tanto, una comunidad de trabajo donde las personas gestionan su conocimiento, aprenden juntas de sus propias prácticas y de sus propias experiencias; donde el aprendizaje de los otros importa de manera capital y donde el 
aprendizaje personal existe y se nutre del conocimiento colectivo.

Una escuela que aprende fomenta una educación de calidad para la vida y esto nos conduce al pensamiento expuesto por Delors (1996) quien señala que «la educación tiene la misión de permitir a todos sin excepción hacer fructificar todos sus talentos y todas sus capacidades de creación, lo que implica que cada uno pueda responsabilizarse de sí mismo y realizar su proyecto personal» (Delors, 1996:12).

\section{CONCLUSIONES}

Como está señalado en el título de este artículo, la convivencia y el clima escolar son claves en la gestión del conocimiento de los/las estudiantes. Por una parte, el clima escolar es un indicador del aprendizaje de la convivencia y es una condición para la apropiación de los conocimientos, habilidades y actitudes, establecidos en el currículum nacional.

Y por otra, la convivencia escolar no es sinónimo de conducta en la sala de clases, ni la gestión del conocimiento se reduce a la performance administrativa de la escuela, al resultado de los aprendizajes de los/las estudiantes, ni a la evaluación de los profesores/as. La gestión del conocimiento dice relación con la práctica y acción global de los proyectos formativos de una escuela y, por tanto, se refiere más a las decisiones estratégicas del conocimiento y del aprendizaje, que a sus contenidos programáticos y curriculares.

El clima escolar no se asocia exclusivamente con la disciplina y la autoridad, sino más bien con la conformación de un ambiente propicio para enseñar y para aprender, en el que se pueden identificar distintos componentes, entre los cuales se pueden mencionar:

- La calidad de las relaciones e interacciones entre las personas (entre todos los miembros de la comunidad educativa).

- La existencia de actividades planificadas en los diversos espacios formativos (aula, patios, bibliotecas, etcétera).

- Un entorno acogedor (limpio, ordenado, decorado, etcétera).

- La existencia de normas y reglas claras, con una disciplina consensuada y conocida por toda la comunidad educativa.

- La existencia de espacios de participación.

Una escuela, colegio o liceo que tenga un clima de aula seguro, basado en la confianza recíproca entre profesores/as y estudiantes y un clima laboral tranquilo, relajado, democrático y participativo, trans- 
versalmente practicado y difundido por la totalidad de la comunidad educativa, contribuirá a desarrollar una convivencia escolar sana, armónica y pacífica, permitiendo que sus estudiantes gestionen su conocimiento de manera creativa, innovadora, eficaz y eficiente, de tal manera de sortear con éxito los desafíos que les impone la sociedad del conocimiento y de la información.

\section{SANTIAGO (ChILE), AGOSTO 2014}

RECIBIDO: AGOSTO 2014 ACEPTADO: NOVIEMBRE 2014

\section{REFERENCIAS BIBLIOGRÁFICAS}

APPlE, M. y N. KING (1989): «QQué enseñan las escuelas?». En GIMENO SACRISTÁN y PÉREZ: La enseñanza: su teoría y su práctica. Madrid: Akal.

Arón, Ana María y Neva Milic (2004): «Clima escolar». Documento de Valoras UC. P.1 Unesco. Santiago: Editorial Andrés Bello.

Disponible en: www.educarchile.cl.

Ascorra, PAUla; H. Arias y CATAlina GrafF (2003): «La escuela como contexto de contención social y afectiva». Revista Enfoques Educacionales. Disponible en: www.facso.uchile.cl.

BAjoit, GuY (2003): Todo cambia. Análisis sociológico del cambio social y cultural en las sociedades contemporáneas. Santiago: LOM Ediciones.

— y ABRAHAM FRANSSEN (1995): «Les Jeunes dans la Compétition Culturelle». Sociologie d'aujourd'hui. París: PUF.

BOURDIEU, PIERRE (1997): Razones prácticas. España: Anagrama.

BRUNNER, JOSÉ JOAQUÍN (2010): «Lenguaje en el hogar, capital cultural y escuela». V Congreso Internacional de la Lengua Española, sección IV, Lengua y Educación Valparaíso, Chile (paper).

Disponible en: www.brunner.cl.

CASASSUS, JUAN (1993): ¿Debe el Estado ocuparse aún de la educación? Mimeo, Santiago.

CERE (1993): Evaluar el contexto educativo. Documento de Estudio. Vitoria: Ministerio de Educación y Cultura. Gobierno Vasco. España.

CiD PATRICIA et al. (2008): «Agresión y violencia en la escuela como factor de riesgo del aprendizaje escolar». Universidad de Concepción, Facultad de Ciencia y Enfermería, Chile. Disponible en: www.scielo.cl.

COLEMAN, J, S. et al. (1966): «Equality of Educational Opportunity». Washington, DC: U. S. Department of Health, Education \& Welfare. Govermment Printing Office.

CONEJO, RODRIGO y JESÚS REDONDO (2001): «El clima escolar percibido por los alumnos de enseñanza media. Una investigación en algunos liceos 
de la Región Metropolitana». Última Década №15. Valparaíso: Ediciones CIDPA.

DELORS, JACQUES (1996): La educación encierra un tesoro. Santiago: Editorial Universitaria.

Descouvieres, Carlos; Jesús Redondo y K. Rojas (2005): «Eficacia y eficiencia de la enseñanza media chilena desde los datos SIMCE 1994, 1998, 2001». Revista Enfoques Educacionales №7.

DíAZ, J. y A. LEIGHTON (2010): «Incidencia del capital cultural en el rendimiento académico de los alumnos del segundo ciclo básico de la Escuela Virgen de la Candelaria». Tesina para optar al grado de Licenciado en Educación (no publicada). Osorno: Universidad San Sebastián.

DíAZ, M. et al. (2002): «Evaluación del rendimiento académico en la enseñanza superior». Revista de Investigación Educativa, 2(20).

Dubet, FRANCOIS y DANILO MARTUCCELli (1998): En la escuela. Sociología de la experiencia escolar. Buenos Aires: Losada.

GAIRÍN, JOAQUín y SERAFÍN ANTÚNEZ (2008): Organizaciones educativas al servicio de la sociedad. Madrid: Wolters Kluwer.

GIMENO, J. (1992): «Investigación e innovación sobre la gestión pedagógica de los equipos de profesores». En: La gestión pedagógica de la escuela. Santiago: UNESCO/OREALC.

GRISAY, A. (1993): «Le fonctionnement des colleges et ses effets sur les éleves de sixieme et de cinquième». Les Dossiers d'Éducation et de la Formation $\mathrm{N}^{\circ} 32$.

HANUSHEK, ERIC A. y STEVEN RIVKIN (1997): «Understanding the Twentieth-Century Growth in U.S. School Spending». Journal of Human Resources, Vol. 32(1). University of Wisconsin Press.

IANNI, NORBERTO (2003): «La convivencia escolar: una tarea necesaria, posible y compleja». OEI, Monografías Virtuales: ciudadanía, democracia y valores en sociedades $\mathrm{N}^{\circ} 2$. Santiago. Disponible en: www.oei.es

JACKSON, PHILIPS (2001): La vida en las aulas. Madrid: Editorial Morata.

KOHLBERG, LAWRENCE (1976): «Moral Stages and Moralization». Likona de Moral Development and Behavior. New York: Holt, Rinehard \& Winston.

— et al. (1997): La educación moral según Laurence Kohlberg. Barcelona: Gedisa.

MiNISTERIO DE EDUCACIÓN, LEY No20.370 (2009): «Ley general de educación». Santiago: Ministerio de Educación.

MINISTERIO DE EDUCACIÓN, LEY No20536 (2011): «Ley sobre violencia escolar». Santiago: Ministerio de Educación.

MAGENDZO, ABRAHAM (2006): «Cómo se implementa el currículum oficialministerial en las instituciones educativas: desafíos y obstáculos». Disponible en: http://mt.educarchile.cl.

MENA, MARÍA ISIDORA y ANA MARÍA VALDÉS (2008). «Clima social escolar». Documento Valoras UC. Santiago: Escuela de Psicología, Universidad Católica. Disponible en: http://es.scribd.com. 
MERTZ, CATAlinA (2006): «La prevención de la violencia en las escuelas». Programa Paz Educa. Disponible en: www.pazciudadana.cl.

Milicic, NeVA (2001): Creo en ti: la construcción de la autoestima en el contexto escolar. Santiago: LOM Ediciones.

Disponible en: http://es.scribd.com.

MinEDUC (2011): «Política nacional de convivencia escolar». Santiago: Ministerio de Educación. Disponible en: www.convivenciaescolar.cl.

(2011): «Conviviendo mejor en la escuela y en el liceo». Santiago: Ministerio de Educación ( $3^{a}$ edición). Disponible en: www.mineduc.cl. (2011): «Ley de calidad y equidad de la educación».

Disponible en: www.mineduc.cl.

(2008): «Marco para la buena enseñanza». Santiago: Ministerio de Educación. Disponible en: www.mineduc.cl.

Mizala, Alejandra, Pilar Romanguera y Teresa Reinaga (1999): «Factores que inciden en el rendimiento escolar en Bolivia». Documentos de Trabajo: Serie Economía. Santiago: Centro de Economía Aplicada, Universidad de Chile.

Oliva, ESTABAN, SADY MONTES y LuSViC TORRELlas (2009): «Educación en valores para el fortalecimiento de la convivencia escolar en el contexto educativo». Revista Educare, Vol. 15, №2.

Disponible en: revistas.upel.edu.ve.

PIAGET, JEAN (1934): El criterio moral en el niño. Barcelona: Niño y Dávila. (1964). Seis estudios de psicología. Editorial Labor.

REDONDO, JESÚS et al. (2009): «Juventud y enseñanza media en Chile del Bicentenario, antecedentes de la Revolución Pingüina».

Disponible en: www.opech.cl

RoDríGUEZ, DARÍo (2004): Diagnóstico organizacional. Santiago: Ediciones Universidad Católica. Disponible en: http://valoras.uc.cl.

Romagnoli, C. y G. GALLARDO (1999): «Alianza efectiva familia y escuela». Documento Valoras UC. Santiago: Escuela de Psicología, Universidad Católica. Disponible en: www.mineduc.cl.

SANTOYO, CÉSAR (2005): «Los valores en la educación».

Disponible en: www.quadernsdigitals.net.

Simon, B. SidneY; HowARd KirSCHENBAUM y Leland W. Howe (1995): Values Clarification, A Practical Action-Directed Handbook. Grand Central Publishing.

STENHOUSE, LAWRENCE (1997): Cultura y educación. Sevilla: Kikirikí.

UNICEF (2005): «Educación de calidad para nuestros hijos. Guía de apoyo para directoras y directores». Disponible en: www.unicef.cl.

VaIL, KathleEN (2005): «Create Great School Climate». The Education Digest 71(4).

VygotSKY, LEV (1988): El desarrollo de los procesos psicológicos superiores. México: Editorial Crítica, Grupo Editorial Grijalbo. 